\title{
Successfully Treated Case of Cholangiolocellular Carcinoma with a Poor Hepatic Functional Reserve Reporting with Various Imaging Findings
}

\author{
Satoshi Takakusagi ${ }^{1}$, Yozo Yokoyama ${ }^{1}$, Kazuko Kizawa ${ }^{1}$, Kyoko Marubashi ${ }^{1}$, \\ Takashi Kosone ${ }^{1}$, Ken Sato ${ }^{2}$, Satoru Kakizaki ${ }^{2,3}$, Kenichi Harada ${ }^{4}$, \\ Hitoshi Takagi ${ }^{1}$ and Toshio Uraoka ${ }^{2}$
}

\begin{abstract}
:
Cholangiolocellular carcinoma (CoCC) is a rare primary liver cancer that is difficult diagnose due to a lack of specific imaging findings. We herein report a case of CoCC accompanied by severe alcoholic cirrhosis. Dynamic computed tomography showed a low-density tumor with a faint surrounding enhancement. Gadolinium-ethoxybenzyl-diethylenetriamine pentaacetic acid-enhanced magnetic resonance imaging revealed iso-intensity in the hepatobiliary phase and a maximum tumor diameter of $53 \mathrm{~mm} .{ }^{18} \mathrm{~F}$-fluoro-2-deoxyglucose position-emission tomography was moderately positive (maximum standardized uptake value: 4.3 ). CoCC was diagnosed based on the pathological findings, including immunohistochemistry. We discuss the diagnostic imaging findings and review previous reports.
\end{abstract}

Key words: cholangiolocellular carcinoma, liver cirrhosis, needle biopsy, immunohistochemistry, EMA, FDG-PET

(Intern Med 60: 873-881, 2021)

(DOI: 10.2169/internalmedicine.5891-20)

\section{Introduction}

Cholangiolocellular carcinoma (CoCC) is an uncommon form of liver cancer with a reported incidence among resected primary liver cancer of $0.56 \%$ (1). CoCC was first reported by Steiner and Higginson in 1959, who determined that it originated from cholangioles or the canals of Hering (2). Recent advancements in the understanding of hepatic progenitor cells (HPCs), which have been reported to exist in the canals of Hering, have suggested that CoCC originates from these cells (3), and this tumor has been categorized as combined hepatocellular-cholangiocarcinoma with stem-cell features in the latest version of the World Health Organization classification of tumors of the digestive system 2010 (4). The imaging diagnosis of CoCC has not been fully established, and it is sometimes difficult to determine a precise diagnosis of CoCC based on a tumor biopsy because of its rarity and similarity to hepatocellular carcinoma (HCC) or intrahepatic cholangiocarcinoma (ICC).

Regarding the postoperative results of surgically treated CoCC, excellent outcomes have been reported with a 5-year recurrence-free survival rate of $41 \%$ and a 5 -year overall survival rate of $72 \%$ (5). Thus, the first-line treatment for CoCC should be surgical resection; however, this may be difficult because CoCC usually develops in patients with chronic liver disease $(5,6)$.

We herein report a case of CoCC that was successfully treated by transcatheter arterial chemoembolization (TACE) and percutaneous radiofrequency ablation (PRFA). We describe the diagnostic imaging findings, including those of ${ }^{18} \mathrm{~F}$-fluoro-2-deoxyglucose position-emission tomography

\footnotetext{
${ }^{1}$ Department of Gastroenterology and Hepatology, Kusunoki Hospital, Japan, ${ }^{2}$ Department of Gastroenterology and Hepatology, Gunma University Graduate School of Medicine, Japan, ${ }^{3}$ Department of Clinical Research, National Hospital Organization Takasaki General Medical Center, Japan and ${ }^{4}$ Department of Human Pathology, Kanazawa University Graduate School of Medicine, Japan Received: July 20, 2020; Accepted: August 29, 2020; Advance Publication by J-STAGE: October 14, 2020 Correspondence to Dr. Satoru Kakizaki, kakizaki@gunma-u.ac.jp
} 
Table 1. Laboratory Data at the First Visit.

\begin{tabular}{|c|c|c|c|}
\hline \multicolumn{2}{|c|}{ Peripheral blood } & \multicolumn{2}{|c|}{ Virus markers } \\
\hline WBC & $3,760 / \mu \mathrm{L}$ & HBs-Ag & $(-)$ \\
\hline $\mathrm{RBC}$ & $372 \times 10^{4} / \mu \mathrm{L}$ & $\mathrm{HCV}-\mathrm{Ab}$ & $(-)$ \\
\hline $\mathrm{Hb}$ & $14.7 \mathrm{~g} / \mathrm{dL}$ & & \\
\hline Platelet count & $54 \times 10^{9} / \mathrm{L}$ & & \\
\hline \multicolumn{2}{|l|}{ Coagulation } & \multicolumn{2}{|c|}{ Liver fibrosis markers } \\
\hline PT activity & $66.7 \%$ & M2BPGi & $8.86 \mathrm{COI}$ \\
\hline PT-INR & 1.27 & FIB-4 index & 15.04 \\
\hline \multicolumn{2}{|c|}{ Blood chemistry } & \multicolumn{2}{|c|}{ Tumor markers } \\
\hline Total protein & $8.1 \mathrm{~g} / \mathrm{dL}$ & AFP & $11.6 \mathrm{ng} / \mathrm{mL}$ \\
\hline Alb & $2.8 \mathrm{~g} / \mathrm{dL}$ & L3 & $13.4 \%$ \\
\hline T-Bil & $2.0 \mathrm{mg} / \mathrm{dL}$ & DCP & $24.7 \mathrm{mAU} / \mathrm{mL}$ \\
\hline D-Bil & $0.7 \mathrm{mg} / \mathrm{dL}$ & CEA & $8.36 \mathrm{ng} / \mathrm{mL}$ \\
\hline AST & $61 \mathrm{IU} / \mathrm{L}$ & CA19-9 & $<2 \mathrm{U} / \mathrm{mL}$ \\
\hline ALT & $21 \mathrm{IU} / \mathrm{L}$ & & \\
\hline LDH & $270 \mathrm{IU} / \mathrm{L}$ & & \\
\hline ALP & $244 \mathrm{IU} / \mathrm{L}$ & & \\
\hline$\gamma$-GT & $347 \mathrm{IU} / \mathrm{L}$ & & \\
\hline BUN & $11.8 \mathrm{mg} / \mathrm{dL}$ & & \\
\hline $\mathrm{Cr}$ & $0.75 \mathrm{mg} / \mathrm{dL}$ & & \\
\hline eGFR & $81.7 \mathrm{~mL} / \mathrm{min} / 1.73 \mathrm{~m}^{2}$ & & \\
\hline $\mathrm{Na}$ & $139 \mathrm{mEq} / \mathrm{L}$ & & \\
\hline $\mathrm{K}$ & $3.8 \mathrm{mEq} / \mathrm{L}$ & & \\
\hline $\mathrm{Cl}$ & $104 \mathrm{mEq} / \mathrm{L}$ & & \\
\hline $\mathrm{HbAlc}$ & $6.2 \%$ & & \\
\hline
\end{tabular}

$\gamma$-GT: $\gamma$-glutamyltransferase, AFP: $\alpha$-fetoprotein, Alb: albumin, ALP: alkaline phosphatase, ALT: alanine aminotransferase, AST: aspartate aminotransferase, BUN: blood urea nitrogen, CA19-9: carbohydrate antigen 19-9, CEA: carcinoembryonic antigen, Cr: creatinine, D-Bil: direct bilirubin, DCP: des- $\gamma$-carboxy prothrombin, eGFR: estimated glomerular filtration rate, FIB-4 index: fibrosis-4 index, $\mathrm{Hb}$ : hemoglobin, HbAlc: hemoglobin A1c, HBs-Ag: hepatitis B surface antigen, HCV-Ab: hepatitis C virus antibody, INR: international normalized ratio, $\mathrm{LDH}$ : lactate dehydrogenase, M2BPGi: mac-2 binding protein glycosylation isomer, PT: prothrombin, RBC: red blood cell count, T-Bil: total bilirubin, WBC: white blood cell count

(FDG-PET), and review previous reports on CoCC.

\section{Case Report}

A 71-year-old man was referred to our hospital because of a liver tumor. He had been diagnosed with alcoholic liver cirrhosis with ascites at another hospital four years previously but had continued to drink. At the first visit to our hospital, he had been consuming at least $90 \mathrm{~g} /$ day of alcohol for more than 30 years. Furthermore, he had suffered from diabetes mellitus and been treated with insulin. Although physical examinations showed mild jaundice in the conjunctivae and edema in the lower legs, his consciousness was clear, and no flapping tremor was observed. No objective abnormalities were observed in the chest or abdomen.

Laboratory examinations (Table 1) showed a decreased white blood cell count and platelet count, reduced prothrombin activity, and decreased serum albumin. Serum total bilirubin, aspartate transaminase, lactate dehydrogenase, and $\gamma$-glutamyltransferase levels were elevated. The levels of alpha-fetoprotein, its subtype L3, and carcinoembryonic antigen were mildly elevated, but the des- $\gamma$-carboxy prothrombin and carbohydrate antigen 19-9 levels were within their normal ranges. The patient was negative for both hepatitis $\mathrm{B}$ surface antigen and hepatitis $\mathrm{C}$ virus antibodies. As liver fibrosis markers, both the mac- 2 binding protein glycosylation isomer level and fibrosis-4 index were quite elevated, implying advanced liver cirrhosis.

Dynamic contrast-enhanced computed tomography (CT) revealed a liver tumor of $53 \mathrm{~mm}$ maximum diameter in the posterior-superior segment. The tumor was slightly enhanced in the arterial phase (Fig. 1A) and showed prolonged enhancement both in the portal phase and late phase (Fig. 1B, C). On ultrasonography (US), the tumor was heterogeneous with an unclear border (Fig. 2A). On plain magnetic resonance imaging (MRI), the liver tumor showed hypo-intensity on T1-weighted imaging (Fig. 1D). On gadolinium-ethoxybenzyl-diethylenetriamine pentaacetic acid 


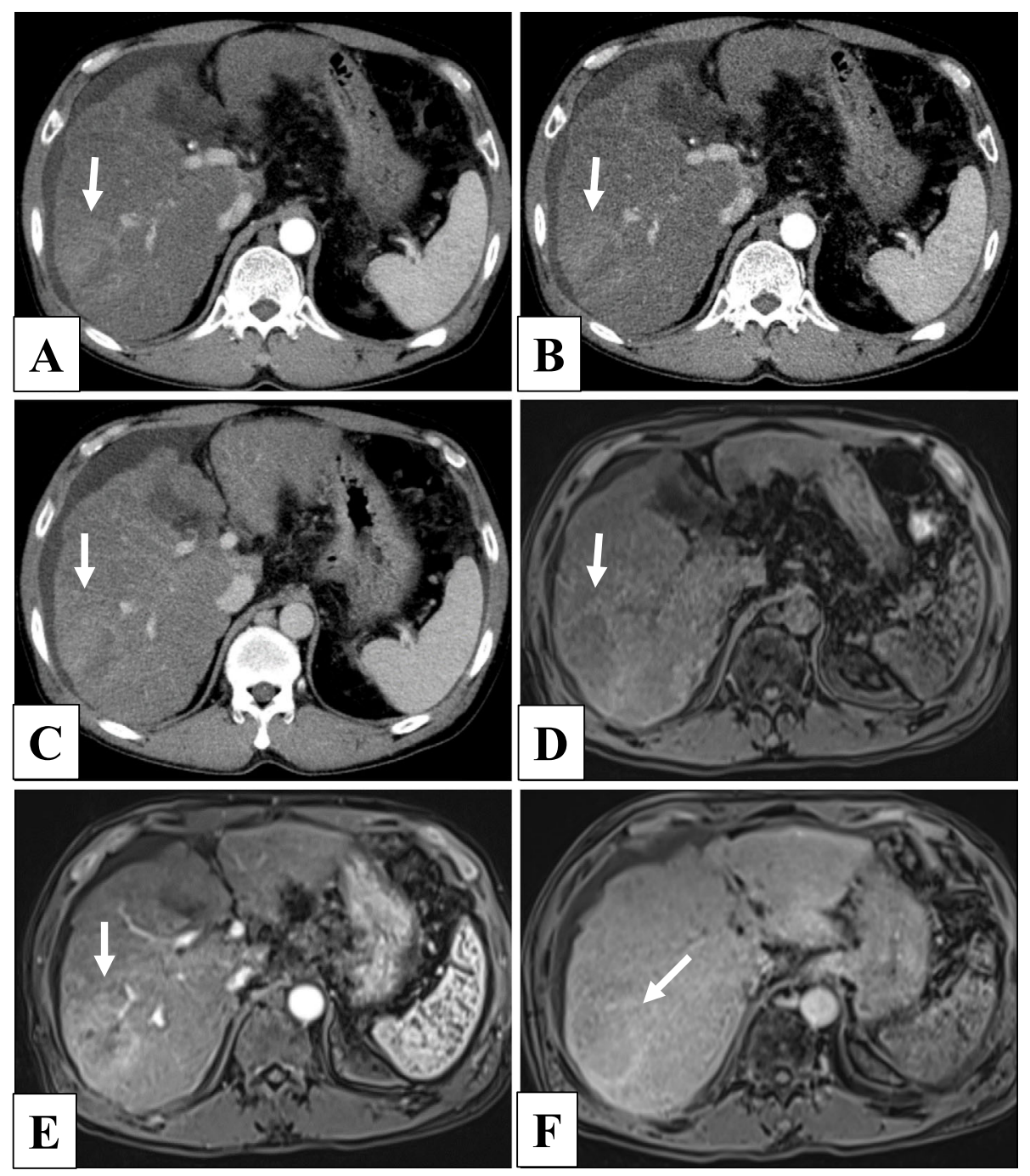

Figure 1. The imaging findings before transcatheter arterial chemoembolization (TACE) and the first percutaneous radiofrequency ablation (PRFA). Dynamic contrast-enhanced computed tomography (CT) revealed a liver tumor with a maximum diameter of $53 \mathrm{~mm}$ in the posterior-superior segment. The tumor was slightly enhanced in the arterial phase (A) and showed prolonged enhancement in both the portal phase (B) and delayed phase (C). On plain MRI, the liver tumor showed hypo-intensity on T1-weighted imaging (D). On gadolinium-ethoxybenzyl-diethylenetriamine pentaacetic acid (Gd-EOB-DTPA)-enhanced MRI, the liver tumor showed slight enhancement in the arterial phase (E) and iso-intensity in the hepatobiliary phase (F).

(Gd-EOB-DTPA)-enhanced MRI, the liver tumor showed slight enhancement in the arterial phase (Fig. 1E) and isointensity in the hepatobiliary phase (Fig. 1F).

Prior to the diagnostic procedure for the primary liver tumor, the tumor could not be conclusively diagnosed as HCC or ICC, and the finding of iso-intensity in the hepatobiliary phase suggested the possible existence of Kupffer cells in the tumor. To determine the treatment, we performed FDGPET. The uptake of FDG in the liver tumor was observed (maximum standardized uptake value: 4.3) without any other hot spots (Fig. 3). Because these imaging findings were not sufficient to make a precise diagnosis, a needle tumor biopsy was performed after controlling the ascites with diuretics. We first diagnosed the needle biopsy specimen as HCC with a pseudo-glandular pattern. We decided to treat him with TACE and PRFA because his tumor met the up-toseven criteria (7) and the hepatic functional reserve was considered insufficient for surgical resection. On angiography, the liver tumor was slightly enhanced (Fig. 4), and TACE was performed with $60 \mathrm{mg}$ of miriplatin hydrate, $3 \mathrm{~mL}$ of lipiodol, and gelatin particle solution. On plain CT performed one day after TACE, the accumulation of pale lipiodol in the tumor was observed (Fig. 5A). Because the lipiodol deposition after TACE was slight and the effect of TACE was considered to have been weak, additional USguided PRFA was performed one week after TACE. The VIVA RF generator ${ }^{\circledR}$ system (STARmed, Goyang, South Korea) was used for PRFA. Dynamic contrast-enhanced CT confirmed that the target lesion was within the ablated area (Fig. 5B). 

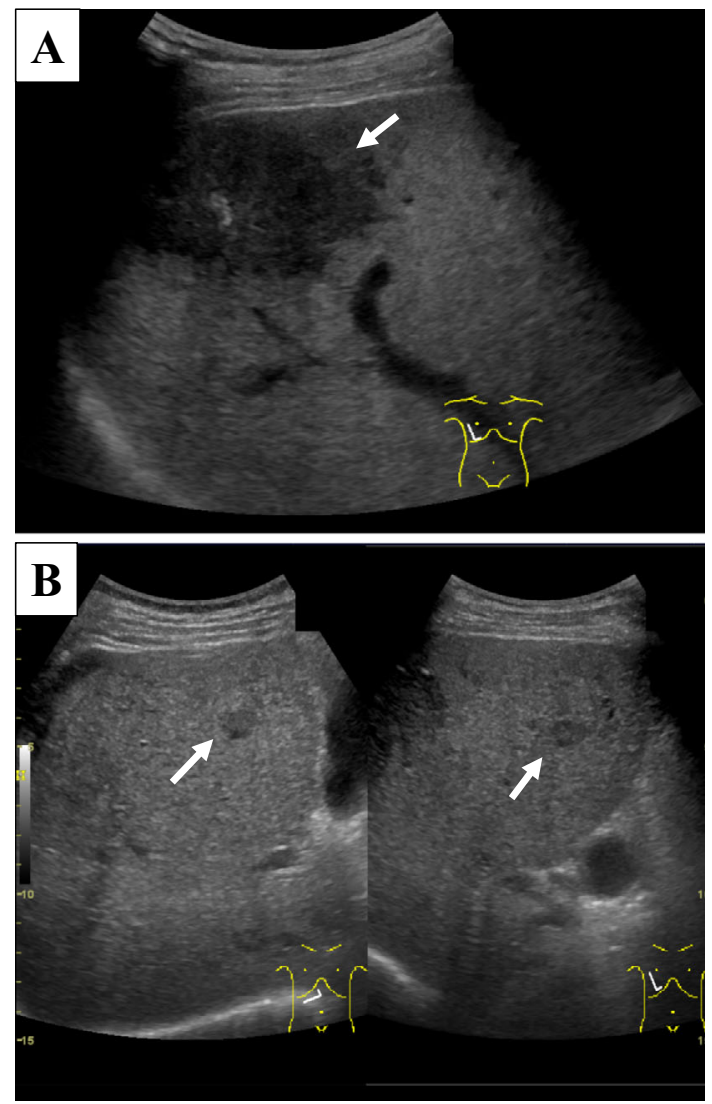

Figure 2. The findings of ultrasonography (US) before the first and second percutaneous radiofrequency ablation (PRFA) treatments. The tumor of the posterior-superior segment was heterogeneous with an unclear border (A). The new lesion of the anterior-inferior segment had a relatively clear border, unlike the tumor of the posterior-superior segment $(B)$.

Because the findings of diagnostic imaging, including dynamic contrast-enhanced CT and EOB-MRI, were not typical for HCC, we consulted a histology expert, and immunohistochemical analyses were performed. Hematoxylin-eosin staining revealed that the tumor shape was dendritic to cribriform with a background of edematous fibrous tissue (Fig. 6A). Immunohistochemistry revealed the tumor cells to be positive for cytokeratin 19 (CK19) (Fig. 6B), with a membranous pattern of epithelial membrane antigen (EMA) (Fig. 6C), NCAM1/CD56 (Fig. 6D), and organic aniontransporting polypeptide (OATP) 1B3 (Fig. 6E) and negative value for arginase 1 (Fig. 6E). The surrounding hepatocytes were also positive for OATP1B3 (Fig. 6F). According to these findings, the pathological diagnosis was $\mathrm{CoCC}$.

Dynamic contrast-enhanced CT performed 3 months after PRFA revealed a slightly enhanced area adjacent to the ablated area and a new lesion $10 \mathrm{~mm}$ in size in the anteriorinferior segment (Fig. 5C). Both lesions showed prolonged enhancement in the portal phase (Fig. 5D) and iso-density in the late phase (Fig. 5E). On US, although both lesions were observed as masses, the new lesion of the anterior-inferior segment had a relatively clear border (Fig. 2B), unlike the lesion of the posterior-superior segment. PRFA of the local
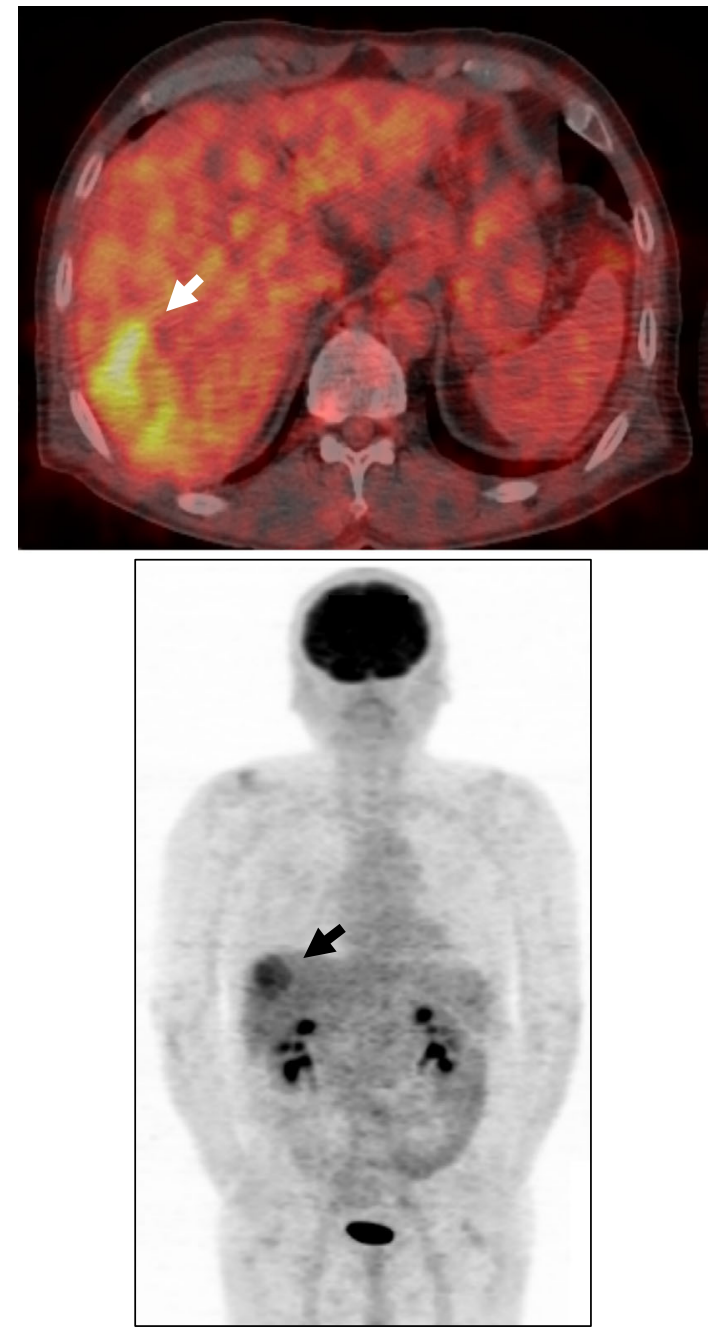

Figure 3. The findings of ${ }^{18} \mathrm{~F}$-fluoro-2-deoxyglucose (FDG) position-emission tomography (Upper: transverse section, Lower: coronal section). The uptake of FDG (arrow) was observed in the liver tumor (maximum standardized uptake value: 4.3 ). There were no other hot spots.

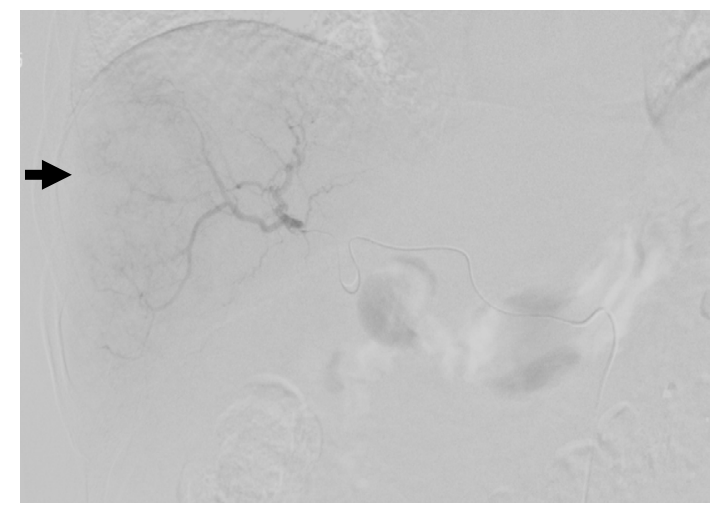

Figure 4. The findings of angiography before the first percutaneous radiofrequency ablation. The liver tumor was slightly enhanced.

recurrence of posterior-superior segment and the new lesion of the anterior-inferior segment was performed. Dynamic contrast-enhanced CT performed three days after PRFA 

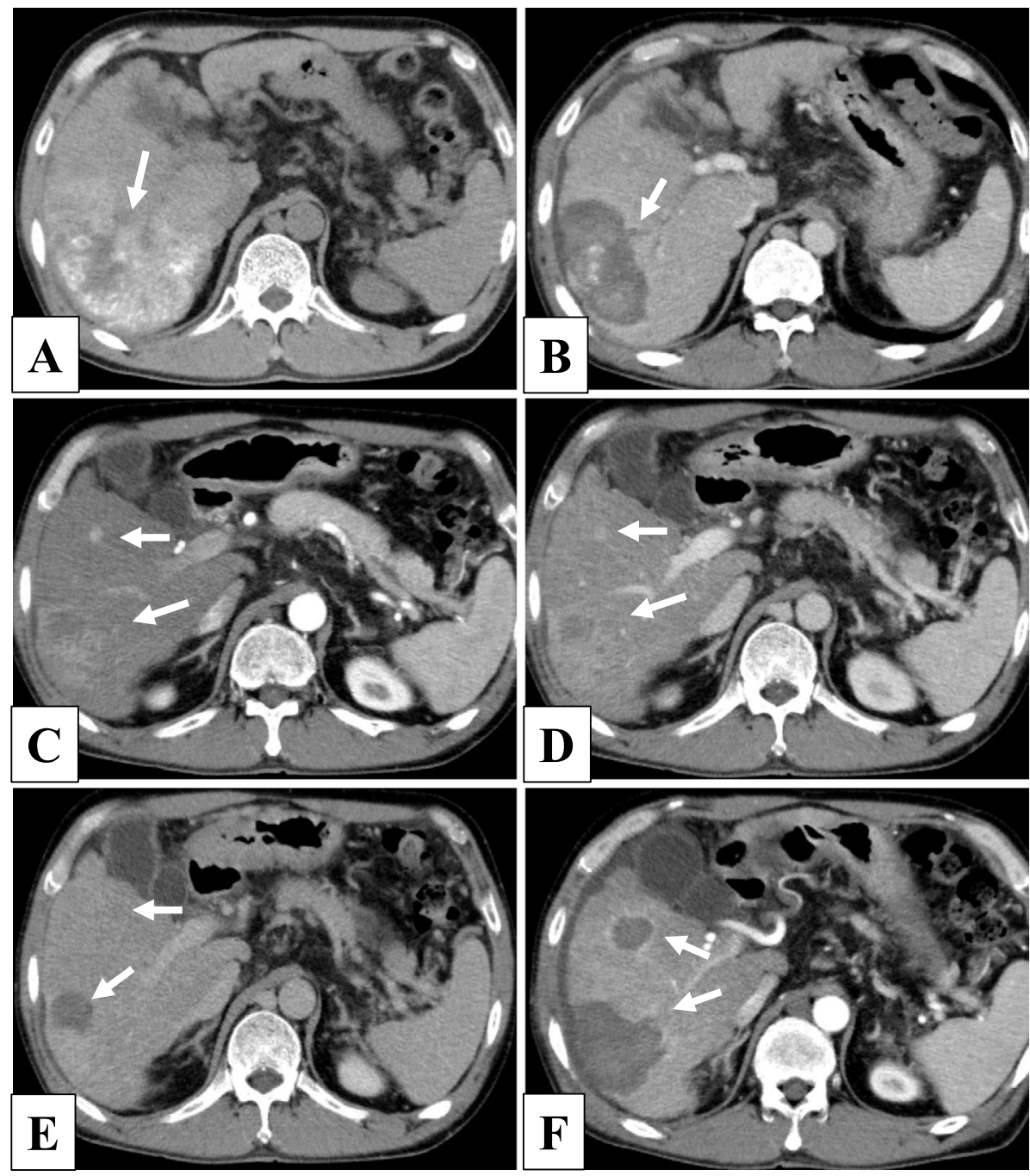

Figure 5. The course of computed tomography (CT) findings after transcatheter arterial chemoembolization (TACE). On plain CT taken one day after TACE, the accumulation of pale lipiodol in the tumor was observed (A). Dynamic contrast-enhanced CT performed after the first PRFA showed that the target tumor was included within the ablation area (B). Dynamic contrast-enhanced CT performed three months after PRFA revealed a slightly enhanced area adjacent to the previously ablated area in the arterial phase. Furthermore, the new lesion was detected in the anterior-inferior segment (C). Both lesions showed prolonged enhancement in the portal phase (D) and iso-density in the late phase (E). Dynamic contrast-enhanced CT performed after the second PRFA treatment showed that both target lesions were included within the ablated area $(\mathrm{F})$.

showed that both target lesions had become unenhanced areas with a safe margin (Fig. 5F). There was no recurrence of $\mathrm{CoCC}$ as of six months later. The clinical course of the patient is presented in Fig. 7.

\section{Discussion}

The major findings from this case report are as follows: (i) CoCC developed in a patient with alcoholic liver cirrhosis; (ii) the tumor showed atypical findings on Gd-EOBDTPA-enhanced MRI, including no defect in the hepatobiliary phase; (iii) FDG-PET showed an increased uptake within the tumor; and (iv) PRFA was tolerable and may be effective, even in patients with a large CoCC and a poor hepatic functional reserve.
$\mathrm{CoCC}$ is very rare malignant liver tumor (1). Although CoCC sometimes develops in patients with chronic liver disease, the etiology is mainly viral hepatitis, and cases involving the development of CoCC in a background of alcoholic liver disease are relatively rare $(5,6)$. Arrizumi et al. (5) analyzed 29 cases of CoCC, and 22 (75.9\%) were complicated with chronic hepatitis or cirrhosis. Nineteen of those 22 cases $(86.4 \%)$ were due to viral hepatitis, while alcoholic liver injury was only responsible in 1 case (4.5\%) (5). Furthermore, many cases have been diagnosed based on the pathological examination of specimens obtained by surgical resection $(5,6)$. Thus, the present case was thought to be valuable given that CoCC developed in a background of alcoholic cirrhosis and was diagnosed by a needle biopsy.

CoCC sometimes contains an HCC and/or ICC compo- 


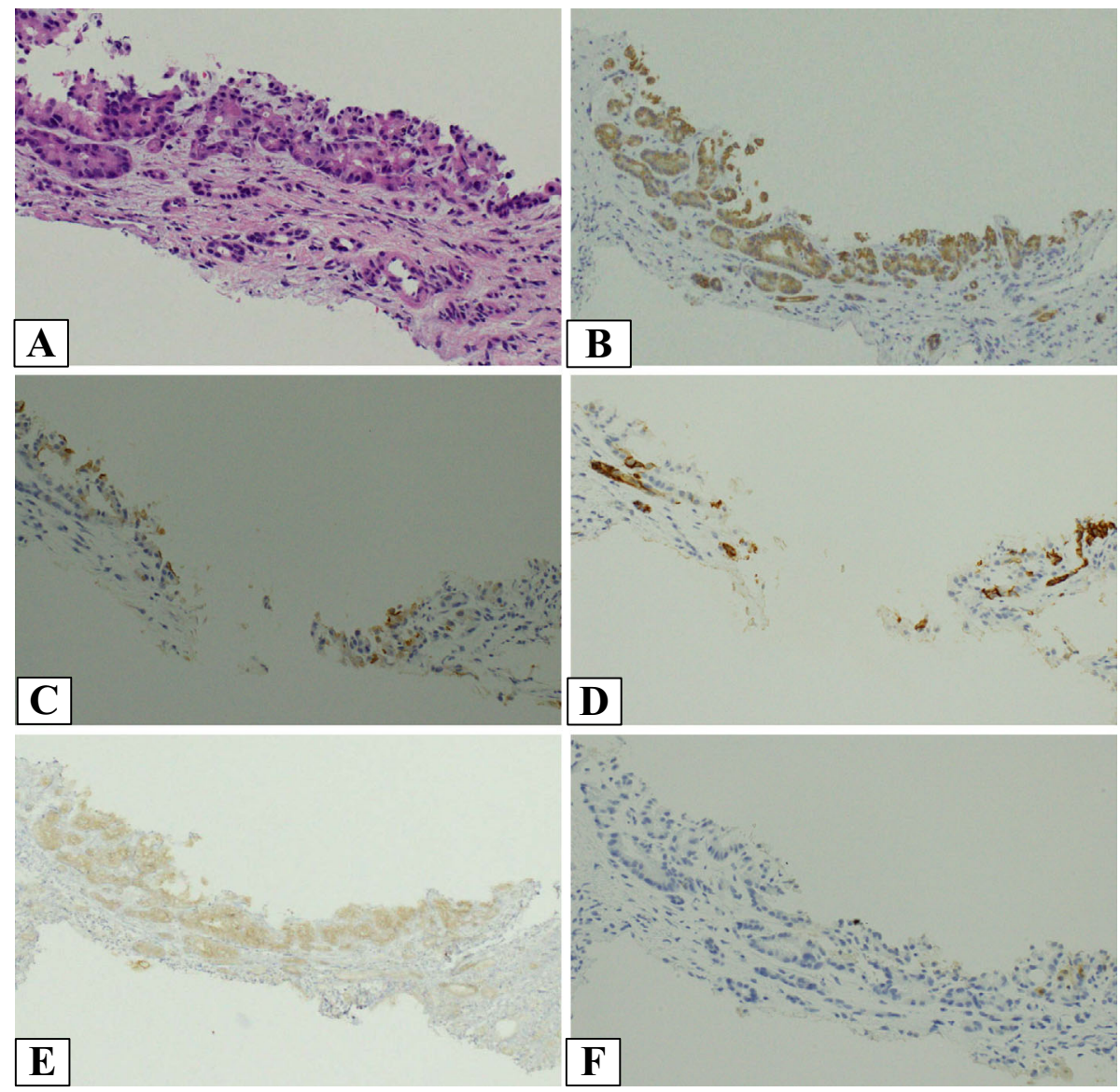

Figure 6. The pathological findings of the tumor of posterior-superior segment before the treatments. The tumor shape was dendritic to cribriform with a background of edematous fibrous tissue, and no mucus secretion by the tumor cells was observed (A). On immunohistochemistry, cytokeratin 19 (CK19) (B), a membranous pattern of epithelial membrane antigen (EMA) (C), and NCAM1/CD56 (D) were detected; the cells were negative for arginase $1(\mathrm{E})$. The tumor cells were positive for organic anion-transporting polypeptide $1 B 3($ OATP1B3) (F). Hematoxylin and Eosin staining $(\times 200)$ (A). CK19 staining $(\times 100)($ B). EMA staining $(\times 400)($ C). NCAM1/CD56 staining $(\times 400)(D)$. Arginase 1 staining $(\times 100)($ E). OATP1B3 staining $(\times 100)(F)$.

nent within the tumor because the origin may be HPC (3). Although the diagnosis of CoCC by imaging modalities, such as CT or MRI, is sometimes difficult, the accumulation of new case reports of $\mathrm{CoCC}$ in recent years has allowed its characterization on imaging. On US, CoCC is generally observed as a heterogeneous hypoechoic lesion with an unclear border (8). The space-occupying lesion often shows hypervascular enhancement from the interior or periphery to the entire tumor, without fast wash-out in the arterial-portal dominant phase, and a distinct defect in the post-vascular phase on contrast-enhanced US with sonazoid $(8,9)$. On MRI, CoCC mainly shows low intensity on T1-weighted imaging, high intensity on T2-weighted imaging, and high intensity on diffusion-weighted imaging $(8,10)$. On dynamic CT or MRI, CoCC generally shows hyper-enhancement of the periphery or the entire lesion in the early phase and hyper-iso-enhancement in the late phase $(8,11,12)$. In addition, CoCC is mostly observed as a clear defect in the hepatobiliary phase on Gd-EOB-DTPA-enhanced MRI $(8,10,13)$. The common findings among these modalities include portal venules penetrating the lesion and the absence of dilatation of the peripheral bile ducts $(5,11)$. The prolonged enhancement on dynamic CT or MRI has been reported to be due to abundant fibrosis among tumor nests (10). It has also been reported that the tumor cells of CoCC proliferate as they replace the surrounding normal liver cell cords and that remaining portal venules are present within tumor; thus, the portal venules appear to penetrate the tumor on imaging $(3,5,10,11)$. In the present case, the border of the lesion was unclear on US, and the lesion showed prolonged enhancement on dynamic CT, which was in line with previous reports. However, no defect image was observed in the hepatobiliary phase of Gd-EOB-DTPA-enhanced MRI, a finding that was considered atypical of CoCC.

Immunohistochemistry revealed that the tumor cells were positive for OATP1B3 (Fig. 6F). This was one reason that no defect image was observed on the hepatobiliary phase of Gd-EOB-DTPA-enhanced MRI. Furthermore, the reduction in the liver function was reported to be correlated with the decreased Gd-EOB-DTPA accumulation in the hepatocytes 


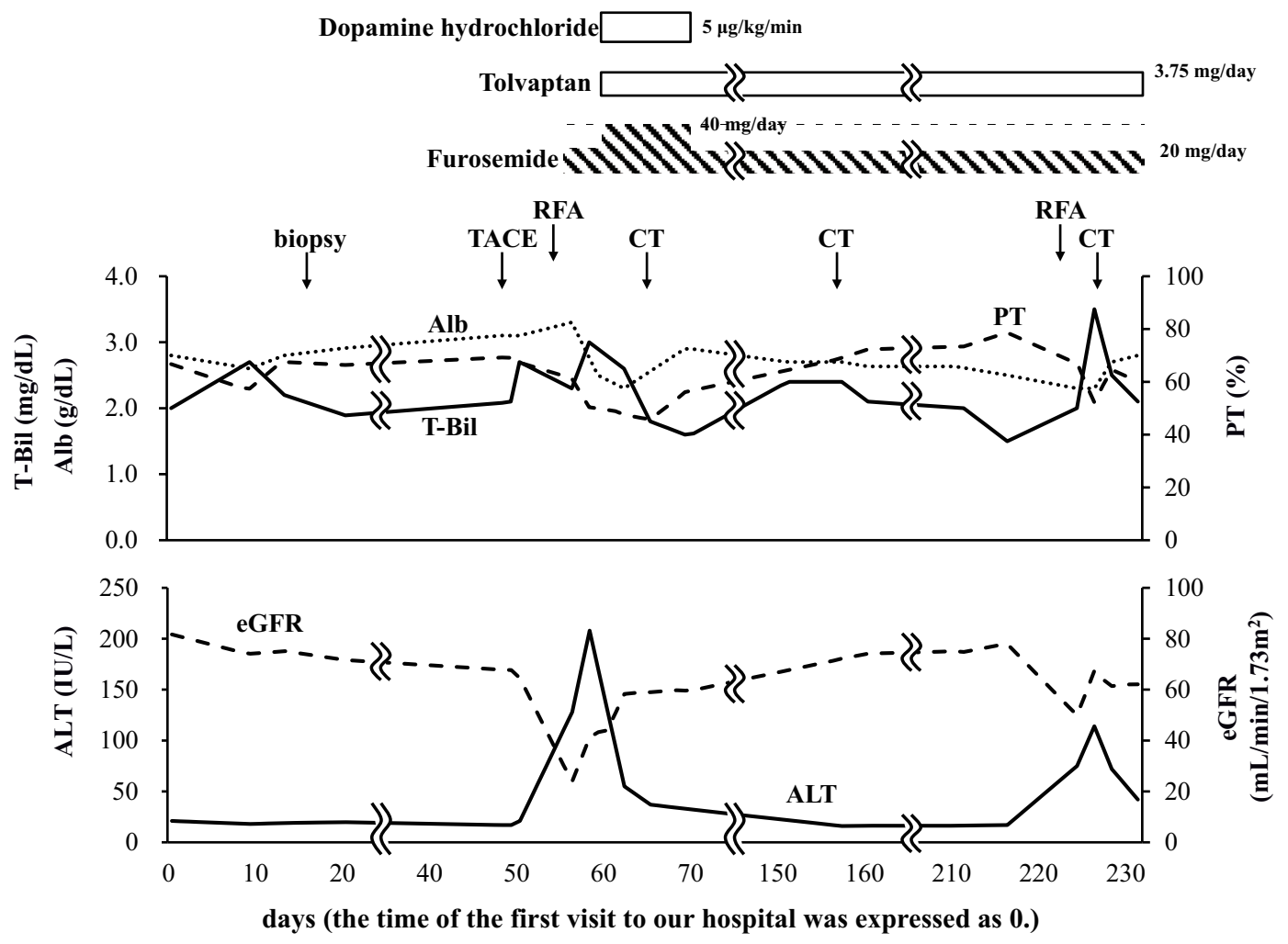

Figure 7. The clinical course of the present case.

Table 2. Reported Cases of CoCC Including FDG-PET Findings.

\begin{tabular}{|c|c|c|c|c|c|c|c|c|c|c|c|c|}
\hline \multirow[b]{2}{*}{ Reference } & \multicolumn{4}{|c|}{ Background } & \multicolumn{4}{|c|}{ Tumor markers } & \multicolumn{4}{|c|}{ FDG-PET } \\
\hline & Age & Sex & Etiology & $\begin{array}{l}\text { Child-Pugh } \\
\text { score }\end{array}$ & $\begin{array}{c}\mathrm{AFP} \\
(\mathrm{ng} / \mathrm{mL})\end{array}$ & $\begin{array}{c}\mathrm{DCP} \\
(\mathrm{mAu} / \mathrm{mL})\end{array}$ & $\begin{array}{c}\text { CEA } \\
(\mathrm{ng} / \mathrm{mL})\end{array}$ & $\begin{array}{l}\text { CA19-9 } \\
\text { (U/mL) }\end{array}$ & $\begin{array}{l}\text { FDG } \\
\text { uptake }\end{array}$ & SUVmax & $\begin{array}{l}\text { Tumor size } \\
\quad(\mathrm{mm})\end{array}$ & Location \\
\hline 11 & 74 & male & $\begin{array}{l}\text { HBs-Ag (-), } \\
\text { HCV-Ab (-) }\end{array}$ & N.D. & normal & normal & normal & 176 & high & 25.2 & 35 & $\begin{array}{l}\text { anterior- } \\
\text { superior } \\
\text { segment }\end{array}$ \\
\hline 13 & 84 & male & $\begin{array}{c}\text { HBs-Ag (-), } \\
\text { HBc-Ab (+), } \\
\text { alcohol }\end{array}$ & N.D. & 5.67 & 25 & 72.89 & 4.9 & no & N.D. & 65 & $\begin{array}{c}\text { medial } \\
\text { segment }\end{array}$ \\
\hline 14 & 59 & male & $\begin{array}{l}\text { HBs-Ag (-), } \\
\text { HBc-Ab (+) }\end{array}$ & 5 & N.D. & N.D. & 53.7 & 6,752 & high & 12.8 & 140 & right lobe \\
\hline Present case & 71 & male & Alcohol & 9 & 11.6 & 24.7 & 8.36 & $<2$ & high & 4.3 & 53 & $\begin{array}{c}\text { posterior- } \\
\text { superior } \\
\text { segment }\end{array}$ \\
\hline
\end{tabular}

AFP: alfa-fetoprotein, CA19-9: carbohydrate antigen 19-9, CEA: carcinoembryonic antigen, CoCC: cholangiolocellular carcinoma, DCP: des- $\gamma$-carboxy prothrombin, FDG-PET: fluoro-2-deoxyglucose position-emission tomography, HBc-Ab: hepatitis B virus core antibody, HBs-Ab: hepatitis B virus surface antigen, HCV-Ab: hepatitis C virus antibody, N.D.: no described, SUVmax: maximun standard uptake value

during the hepatobiliary phase (14). In the present case, the impaired liver function [Child-Pugh score, 8; albuminbilirubin (ALBI) score, -1.37] may have caused the atypical Gd-EOB-DTPA-enhanced MRI findings. While Gd-EOBDTPA-enhanced MRI is useful for detecting liver tumors, including $\mathrm{HCC}$ and $\mathrm{CoCC}$, hepatic dysfunction may affect the qualitative diagnosis of the tumor.

Regarding the origin of $\mathrm{CoCC}$, several hypotheses have been made, including the cholangioles or canals of Hering origin theory (3) and the interlobular bile ducts origin theory (6). In either case, since $\mathrm{CoCC}$ tends to originate from a peripheral side of bile duct, the peripheral bile duct shows no dilatation.

Regarding FDG-PET, aside from our case, only three other cases of CoCC have been reported $(10,15,16)$. In three of the four cases including our own, CoCC showed a high uptake of FDG within the tumor (Table 2). Mori et al. noted that malignant CoCC cells show significant alterations in their metabolism during the process of transformation from normal cells, so the differences in the uptake of FDG by different tumors might be caused by the malignant potential and/or tumor characteristics (10). Although there have been few reports on the FDG uptake in $\mathrm{CoCC}$ and further studies are needed, the disordered carbohydrate metabolism 
Table 3. Reported Cases of CoCC Treared by Minimally Invasive Treatment.

\begin{tabular}{|c|c|c|c|c|c|c|c|c|c|}
\hline \multirow[b]{2}{*}{ Reference } & \multicolumn{4}{|c|}{ Background } & \multicolumn{3}{|c|}{ Tumor } & \multicolumn{2}{|c|}{ Tremtment } \\
\hline & Age & Sex & Etiology & $\begin{array}{l}\text { Child-Pugh } \\
\text { score }\end{array}$ & $\begin{array}{l}\text { Maximum } \\
\text { diameter }\end{array}$ & $\begin{array}{l}\text { Single or } \\
\text { multiple }\end{array}$ & Location & Method & Outcome \\
\hline 20 & 59 & male & Liver cirrhosis & 6 & 30 & Single & Segment 3 & RFA & $6 \mathrm{M}$ alive \\
\hline 21 & 79 & male & $\mathrm{HCV}$ & 6 & 60 & Single & Segment 5 & TACE & $2 \mathrm{Y}$ alive \\
\hline 22 & 71 & male & Alcohol & 7 & 54 & Multiple & $\begin{array}{c}\text { Anterior segment, } \\
\text { segment } 2 \text {, segment } 4\end{array}$ & $\begin{array}{c}\text { TACE, } \\
\text { chemotherapy }\end{array}$ & $1 \mathrm{Y}$ alive \\
\hline 23 & $70 \mathrm{~s}$ & male & $\mathrm{HCV}$ & N.D. & 30 & Single & Segment 4 & TACE & $3 Y$ dead \\
\hline Present case & 71 & male & Alcohol & 9 & 53 & Single & Segment 7 & TACE, RFA & $6 \mathrm{M}$ alive \\
\hline
\end{tabular}

CoCC: cholangiolocellular carcinoma, HCV: hepatitis C virus, N.D.: no described, RFA: radiofrequency ablation, TACE: transcatheter arterial chemoembolization, M: month, Y: year

in CoCC might change during the clinical course.

Immunohistochemistry using a hepatocyte marker (arginase 1), cholangiocyte marker (CK19), stem cell feature (NCAM1/CD56), and EMA is useful for the differential diagnosis among $\mathrm{CoCC}, \mathrm{HCC}$, and $\operatorname{ICC}(6,17-19)$. The present findings were compatible with $\mathrm{CoCC}$. In particular, EMA positivity at the membranous site of the cancer duct (membranous pattern) has been recognized as a specific feature of $\operatorname{CoCC}(17,18)$. In the present case, although the patient was initially pathologically diagnosed with HCC, the immunohistochemical findings and characteristic growth pattern ultimately led us to the definitive diagnosis of CoCC. We are of the opinion that immunohistochemistry, including EMA staining, is necessary for the diagnosis of liver tumors showing atypical imaging findings, especially when the tumor specimen is obtained by a fine-needle biopsy.

Although $\mathrm{CoCC}$ is rare, it was reported that CoCC patients who underwent curative surgical resection had special characteristics with a favorable long-term survival due to this lesion's relatively low-invasive histopathological characteristics (unlike ICC) (5). Furthermore, the case of a patient with CoCC who had repeated recurrence in the remnant liver and was treated by additional surgical resection six times and who achieved a seven-year survival after the first curative surgical resection was also reported (20). Thus, surgical resection is considered the optimal first-line treatment for CoCC, regardless of whether it is performed as the initial treatment or as treatment for recurrence.

However, other treatment methods should also be considered for cases with a poor liver function due to chronic liver disease. To our knowledge, there had been four reports of minimally invasive treatments not associated with surgical resection, except for our present case. The etiologies of CoCC were viral hepatitis in two cases, alcohol in one case, and liver cirrhosis in one case. Minimally invasive treatments were selected because of complications, the tumor size, the number of tumors, or the patient's refusal to undergo an operation (21-24). Although RFA under laparotomy was performed in one case (21), there were no cases treated by PRFA (Table 3). Regarding PRFA, to our knowledge, there is only one case report discussing the effectiveness of
PRFA for local recurrence; surgical resection was performed as the initial treatment and as the treatment for the first recurrence. Thereafter, PRFA was performed 7 times for a total of 10 recurrent lesions, and the patient was able to survive for 9.2 years (25).

In the present case, the lipiodol deposition after TACE was slight, and the effect of TACE was considered weak. As a result, additional PRFA achieved good tumor ablation. Because the tumor was large and the number of PRFA punctures was supposed to be many, we performed PRFA after TACE to decrease the risk of PRFA complications, such as bleeding and tumor dissemination. The local recurrence in our case was mostly due to the margin of ablation being minimal, but recurrent lesions were able to be treated by additional PRFA. We believe that PRFA may be a useful option for CoCC patients with a deteriorated liver functional reserve.

HPC would be activated when the replication of mature epithelial cell compartments of liver, hepatocytes, and/or cholangiocytes are damaged $(26,27)$. Although rare, cases of double primary hepatic cancer consisting of HCC and CoCC have been reported $(28,29)$. HPC may give rise to various types of primary hepatic cancer, including $\mathrm{HCC}$, ICC, CoCC, and combined HCC and ICC, depending on the stage of carcinogenesis (28). Thus, continuous imaging follow-up should be performed to detect not only the recurrence of $\mathrm{CoCC}$ but also occurrence of $\mathrm{HCC}$, ICC, or combined HCC and ICC.

In conclusion, we experienced a case of CoCC accompanied by alcoholic liver cirrhosis that was first diagnosed as HCC and treated by TACE and PRFA. A precise immunohistochemical analysis subsequently revealed CoCC. Local recurrence was also able to be treated by PRFA without serious adverse events. The FDG-PET findings of CoCC have only been reported in four cases of $\mathrm{CoCC}$, including the present case.

The authors state that they have no Conflict of Interest (COI). 


\section{References}

1. Shiota K, Taguchi J, Nakashima O, et al. Clinicopathologic study on cholangiolocellular carcinoma. Oncol Rep 8: 263-268, 2001.

2. Steiner PE, Higginson J. Cholangiolocellular carcinoma of the liver. Cancer 12: 753-759, 1959.

3. Komuta M, Spee B, Vander Borght S, et al. Clinicopathological study on cholangiolocellular carcinoma suggesting hepatic progenitor cell origin. Hepatology 47: 1544-1556, 2008.

4. Bosman FT, Carnerio F, Hyban RH, et al. WHO Classification of Tumors of the Digestive System. International Agency for Research on Cancer, Lyon, 2010.

5. Ariizumi S, Kotera Y, Katagiri S, et al. Long-term survival of patients with cholangiolocellular carcinoma after curative hepatectomy. Ann Surg Oncol 21: S451-458, 2014.

6. Maeno S, Kondo F, Sano K, et al. Morphometric and immunohistochemical study of cholangiolocellular carcinoma: comparison with non-neoplastic cholangiole, interlobular duct and septal duct. J Hepatobiliary Pancreat Sci 19: 289-296, 2012.

7. Bolondi L, Burroughs A, Dufour JF, et al. Heterogeneity of patients with intermediate (BCLC B) Hepatocellular Carcinoma: proposal for a subclassification to facilitate treatment decisions. Semin Liver Dis 32: 348-359, 2012.

8. Osawa M, Saitoh S, Fujiyama S, et al. Cholangiolocellular carcinoma in a young patient who showed sustained virological response after treatment for hepatitis $\mathrm{C}$ virus infection. Intern Med 56: 3033-3040, 2017.

9. Joshita S, Ichijo T, Suzuki F, et al. A case of well-differentiated cholangiolocellular carcinoma visualized with contrast-enhanced ultrasonography using Sonazoid. Hepatol Res 39: 207-212, 2009.

10. Mori N, Ichikawa T, Hashimoto J, et al. Cholangiolocellular carcinoma of the liver exhibiting high F-18 FDG uptake. Tokai J Exp Clin Med 41: 60-64, 2016.

11. Asayama $Y$, Tajima $T$, Okamoto $D$, et al. Imaging of cholangiolocellular carcinoma of the liver. Eur J Radiol 75: e120-e125, 2010.

12. Motosugi U, Ichikawa $\mathrm{T}$, Nakajima $\mathrm{H}$, et al. Cholangiolocellular carcinoma of the liver: imaging findings. J Comput Assist Tomogr 33: 682-688, 2009.

13. Haradome $H$, Unno $T$, Morisaka $H$, et al. Gadoxetic acid disodium-enhanced MR imaging of cholangiolocellular carcinoma of the liver: imaging characteristics and histopathological correlations. Eur Radiol 27: 4461-4471, 2017.

14. Haimerl M, Verloh N, Fellner C, et al. MRI-based estimation of liver function: Gd-EOB-DTPA-enhanced T1 relaxometry of 3T vs. the MELD score. Sci Rep 4: 5621, 2014.

15. Takahashi Y, Sato S, Ishitobi H, et al. Intrahepatic cholangiolocellular and cholangiocellular carcinoma - differences in the ${ }^{18} \mathrm{~F}-\mathrm{FDG}$ PET/CT findings. Intern Med 56: 3027-3031, 2017.

16. Ishii N, Suzuki H, Tsukagoshi M, et al. Giant cholangiolocellular carcinoma with early recurrence that was difficult to distinguish from cholangiocellular carcinoma: report of a case. Int Surg 100: 1111-1116, 2015.

17. Kondo F, Fukusato T. Pathogenesis of cholangiolocellular carcinoma: possibility of an interlobular duct origin. Intern Med 54: 1685-1694, 2015.

18. Nakano M. Pathology of cholangiolocellular carcinoma. Tan To Sui (Biliary Tract and Pancreas) 25: 343-349, 2004 (in Japanese).

19. Komuta M, Govaere O, Vandecaveye V, et al. Histological diversity in cholangiocellular carcinoma reflects the different cholangiocyte phenotypes. Hepatology 55: 1876-1888, 2012.

20. Tomioku M, Yazawa N, Furukawa D, et al. Repeated hepatectomy for recurrent intrahepatic cholangiolocellular carcinoma: report of a case. Tokai J Exp Clin Med 41: 92-96, 2016.

21. Nagata N, Gocho Seiho, Suzuki T, et al. A case of cholangiolocellular carcinoma with diabetes mellitus, chronic renal failure, and liver cirrhosis. Tando (J Japan Biliary Assoc) 22: 669-675, 2008 (in Japanese, Abstract in English).

22. Yokosuga J, Takikawa S, Koike K, et al. A case of cholangilocellular carcinoma diagnosed after liver biopsy and treated with interventional radiology. Kanzo 53: 829-838, 2012 (in Japanese, Abstract in English).

23. Yamaguchi T, Seki T, Inokuchi R, et al. S-1 monotherapy in a patient with cholangiolocellular carcinoma: a case report. Mol Clin Oncol 5: 762-766, 2016.

24. Ishida $T$, Kondo $F$, Tokairin $T$, et al. A case of primary liver cancer with components of so called cholangilocellular carcinoma and intrahepatic cholangicarcinoma. Shindanbyouri 35: 110-117, 2018 (in Japanese).

25. Shinohara K, Ebata T, Yokoyama Y, et al. Successful local treatment for repeated hepatic recurrences of cholangiolocellular carcinoma: a report on a long-term survivor. Surg Case Rep 3: 120, 2017.

26. Roskams TA, Libbrecht L, Desmet VJ. Progenitor cells in diseased human liver. Semin Liver Dis 23: 385-396, 2003.

27. Roskams T, Yang SQ, Koteish A, et al. Oxidative stress and oval cell accumulation in mice and humans with alcoholic and nonalcoholic fatty liver disease. Am J Pathol 163: 1301-1311, 2003.

28. Yamamoto M, Oshita A, Nishisaka T, et al. Synchronous double primary hepatic cancer consisting of hepatocellular carcinoma and cholangiolocellular carcinoma: a case report. J Med Case Rep 12: 224, 2018.

29. Suzumura K, Asano Y, Hirano T, et al. Synchronous double cancers of primary hepatocellular carcinoma and cholangiolocellular carcinoma: a case report. Surg Case Rep 2: 139, 2016.

The Internal Medicine is an Open Access journal distributed under the Creative Commons Attribution-NonCommercial-NoDerivatives 4.0 International License. To view the details of this license, please visit (https://creativecommons.org/licenses/ by-nc-nd/4.0/).

(C) 2021 The Japanese Society of Internal Medicine Intern Med 60: 873-881, 2021 